\title{
Ironing out Their Differences: Dissecting the Structural Determinants of a Phenylalanine Aminomutase and Ammonia Lyase
}

Matthew M. Heberling, ${ }^{\dagger}$ Marcelo F. Masman, ${ }^{\dagger}$ Sebastian Bartsch, ${ }^{\dagger}\|\|$ Gjalt G. Wybenga, ${ }^{\dagger} \perp$ Bauke W. Dijkstra, ${ }^{\ddagger}$ Siewert J. Marrink, ${ }^{\S}$ and Dick B. Janssen ${ }^{*}$,

${ }^{\dagger}$ Department of Biochemistry, Groningen Biomolecular Sciences and Biotechnology Institute, University of Groningen, Nijenborgh 4, 9747 AG, Groningen, The Netherlands

\$Laboratory of Biophysical Chemistry, Groningen Biomolecular Sciences and Biotechnology Institute,

${ }^{\S}$ Department of Molecular Dynamics, Groningen Biomolecular Sciences and Biotechnology Institute, University of Groningen, Nijenborgh 7, 9747 AG, Groningen, The Netherlands

Present addresses:

c-LEcta GmbH, Perlickstraße 5, 04103 Leipzig, Germany

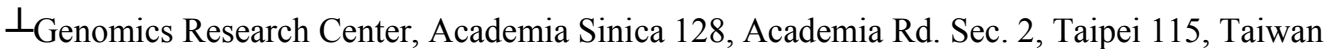

Corresponding Author:

*E-mail: d.b.janssen@rug.nl

\section{SUPPORTING INFORMATION}

\section{Table of Contents}

Figure S1 | Inter-loop salt bridge analysis

Figure S2 | Probing PAM residue position 97 ........................................................................... 2

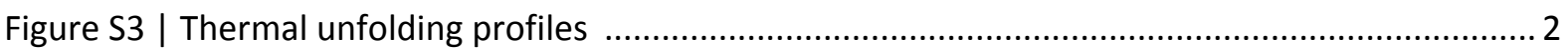

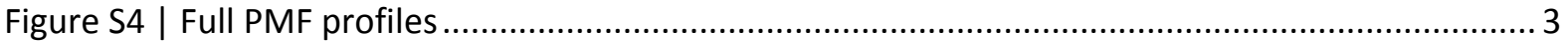

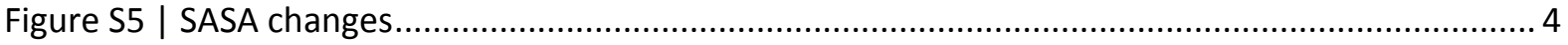

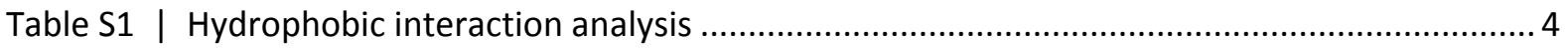

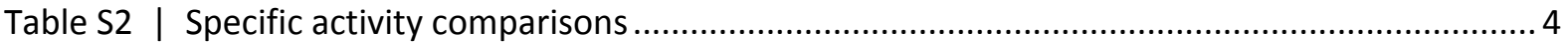

Table S3 | Cosolvent effects on activity ............................................................................... 5

Table S4 | Temperature effects on activity............................................................................ 5

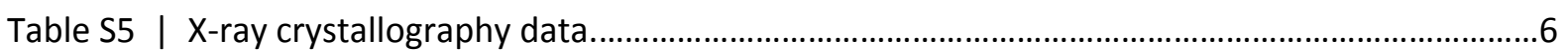

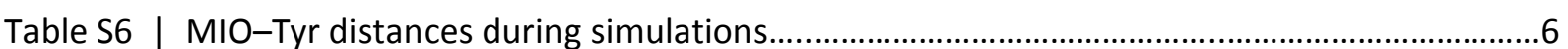

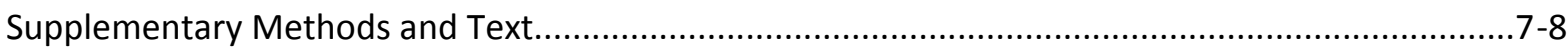

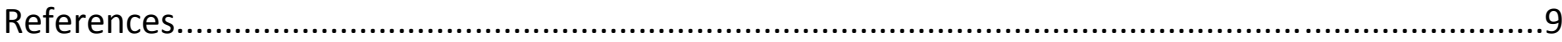



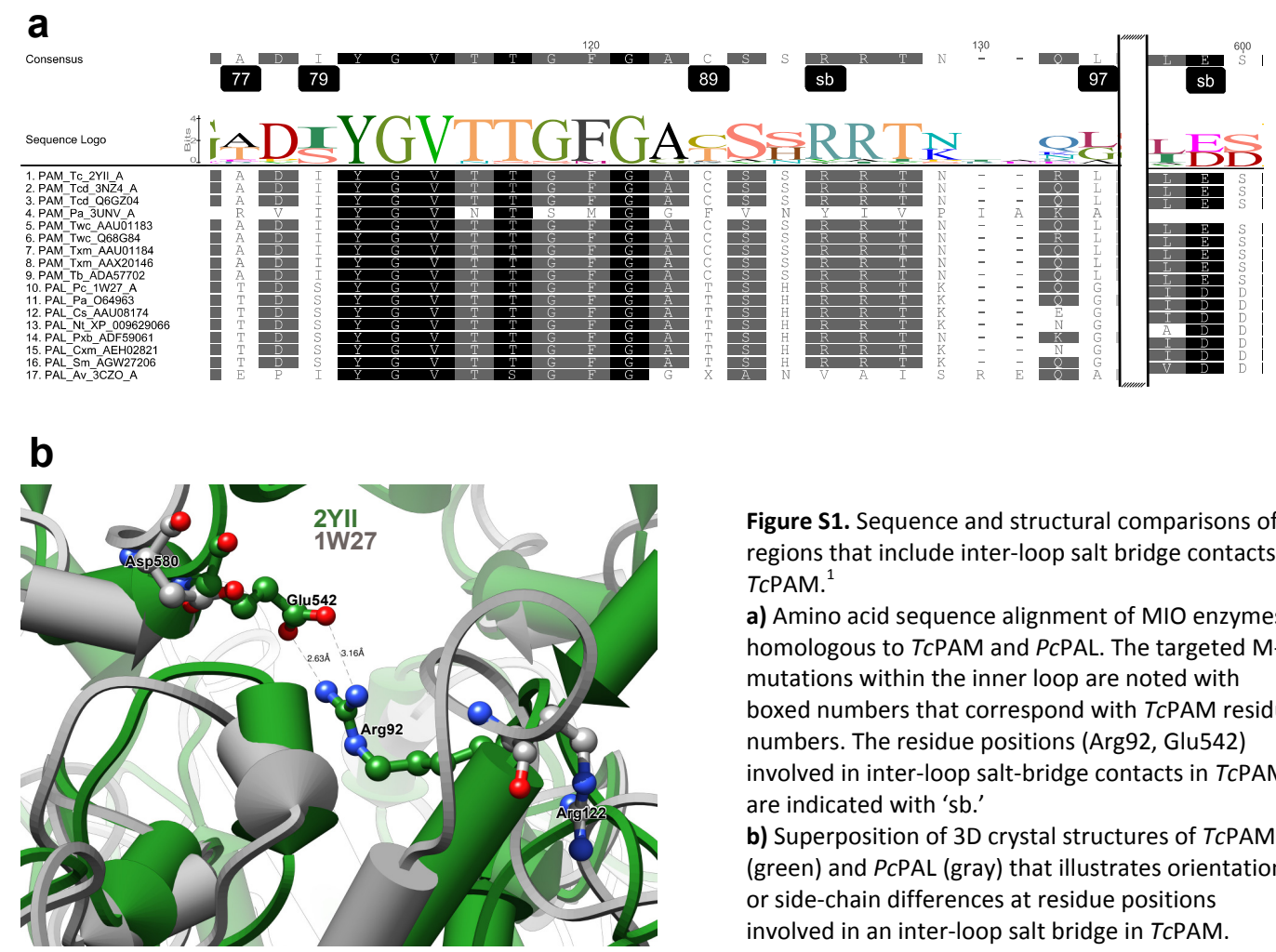

Figure S1. Sequence and structural comparisons of regions that include inter-loop salt bridge contacts in TCPAM. ${ }^{1}$

a) Amino acid sequence alignment of MIO enzymes homologous to TCPAM and PCPAL. The targeted M-L mutations within the inner loop are noted with boxed numbers that correspond with TCPAM residue numbers. The residue positions (Arg92, Glu542) involved in inter-loop salt-bridge contacts in TCPAM are indicated with 'sb.'

b) Superposition of 3D crystal structures of TCPAM (green) and PCPAL (gray) that illustrates orientation or side-chain differences at residue positions involved in an inter-loop salt bridge in TCPAM.

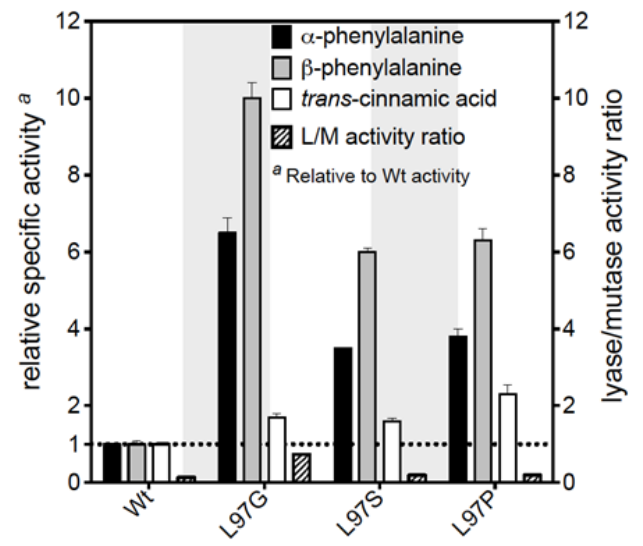

Figure S2. Activity comparisons between Wt PAM and position 97 mutants. Primary y-axis: Specific activity comparisons between Wt PAM and single loop mutants to probe position 97 . Secondary $y$-axis: Lyase/mutase (L/M) activity ratio comparisons with substrate, $(R)-\beta$ phenylalanine.

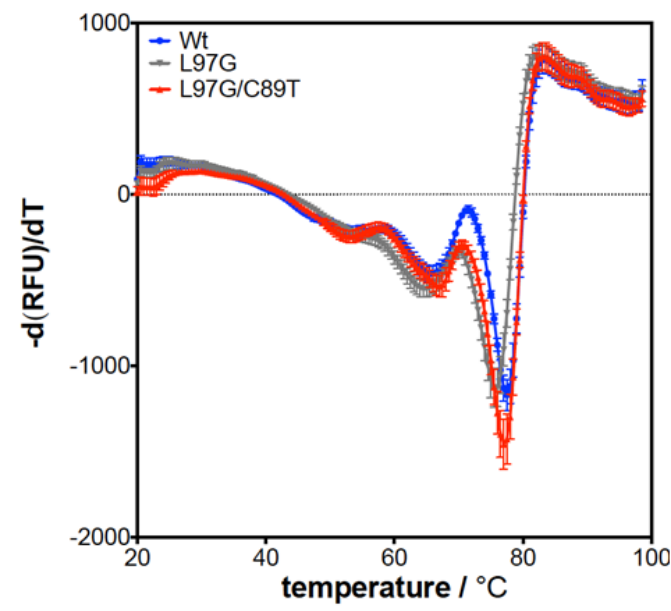

Figure S3. Thermal unfolding profiles of Wt PAM and the relevant loop mutants assayed in the temperature-activity experiments (Figure 4). Each profile represents a mean \pm $\operatorname{SEM}(n=6)$ representing triplicate measurements at two different enzyme concentrations. 
a

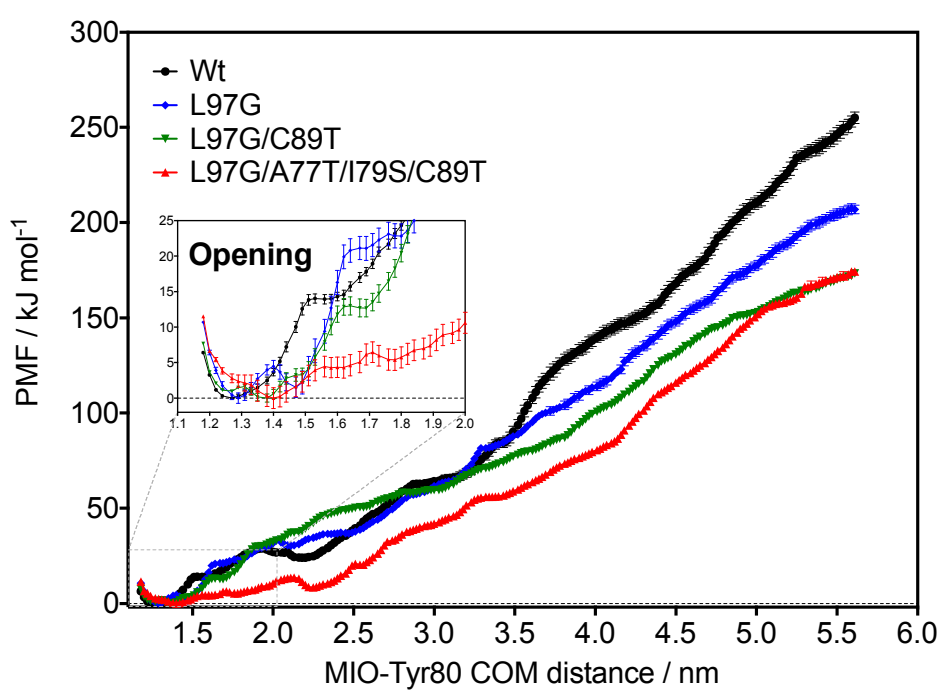

b
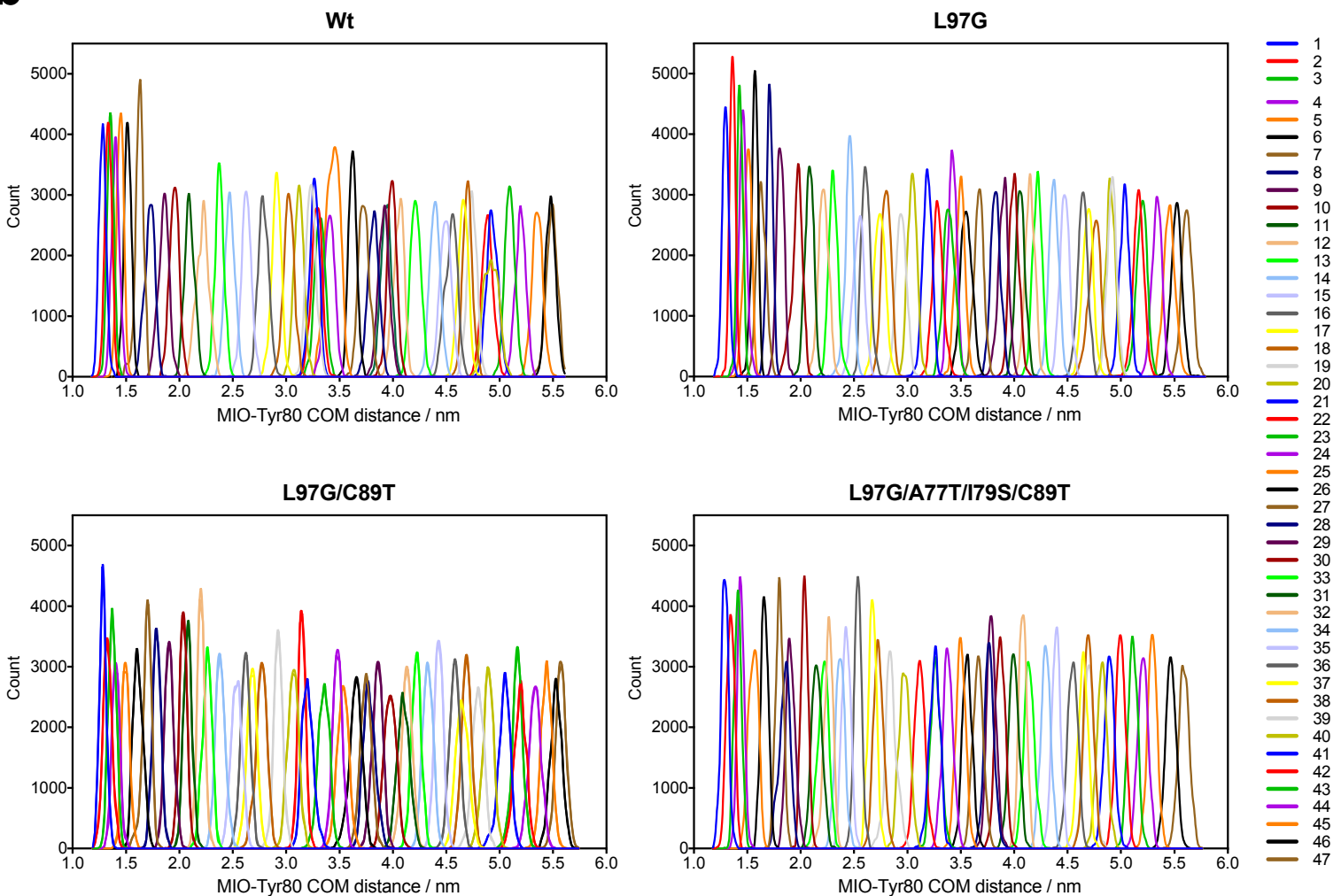

Figure S4. Umbrella sampling simulations. a) Full-range PMF profiles for the umbrella-sampling simulations of the PAM systems in this study. Up to $2 \mathrm{~nm}$ of the MIO-Tyr80 distance was deemed as the loop opening process (inset), whereas beyond $2 \mathrm{~nm}$ was considered a distance where globally-induced unfolding initiated from the pulled inner loop. The full range of pulling/umbrella-sampling simulations covered up to $\sim 5.5 \mathrm{~nm}$. b) Histograms of the configurations within the 47 umbrella sampling windows utilized in this study. Supplementary to Figure $\mathbf{5 b}$. 


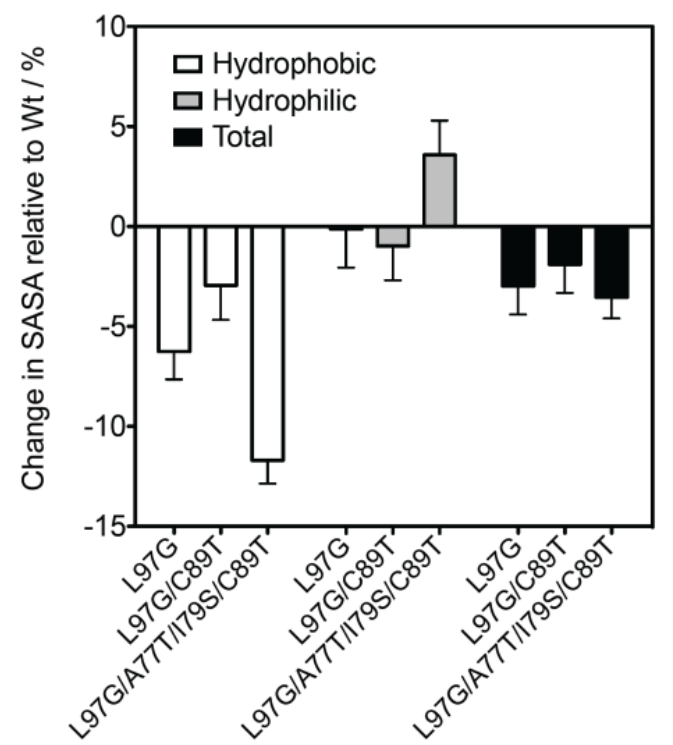

Figure S5. Changes in solvent accessible surface area

(SASA) during resting state simulations of the inner loop for each PAM system. All values are given as a percent change relative to Wt PAM, averaged over time. Supplementary to Figure 5d.

Table S1. Summary of hydrophobic interactions for selected inner loop residues ${ }^{a}$

\begin{tabular}{|c|c|c|c|c|}
\hline $\begin{array}{c}\text { Inner loop } \\
\text { residue }\end{array}$ & Atom & Residue & Atom & $\begin{array}{c}\begin{array}{c}\text { Distance } \\
{[\AA ̊]}\end{array} \\
\end{array}$ \\
\hline \multirow[t]{5}{*}{ Leu97 $^{b}$} & \multirow[t]{3}{*}{$\mathrm{C}_{\delta_{1}}$} & & $c_{\beta}$ & 3.73 \\
\hline & & Glu74 & $C_{\gamma}$ & 4.23 \\
\hline & & Ala223 & $c_{\beta}$ & 4.54 \\
\hline & \multirow{2}{*}{${ }^{C} \delta_{2}$} & & $c_{\beta}$ & \\
\hline & & Val82 & $\mathrm{C}_{\gamma_{1}}$ & 5.13 \\
\hline \multirow[t]{2}{*}{$11 \mathrm{le} 79^{b}$} & $\mathrm{C}_{\delta_{1}}$ & & $\mathrm{C}_{\beta}$ & 3.94 \\
\hline & $c_{\beta}$ & Val82 & $C_{\gamma_{2}}$ & 4.09 \\
\hline
\end{tabular}

Table S2. Specific activity comparisons of Wt TcPAM and loop mutants with substrates. Supplementary to Figure 2.

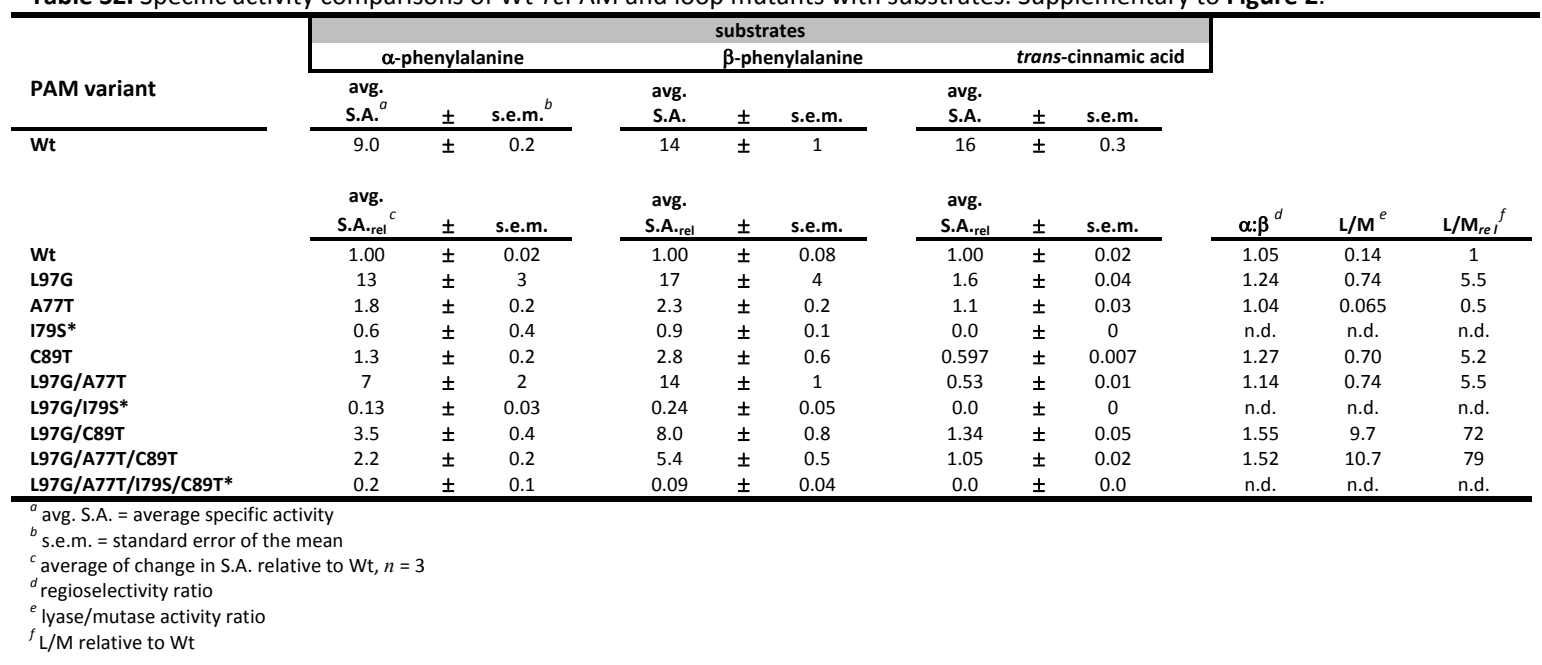


Table S5. X-ray crystallography data collection and refinement statistics.

PAM L97G PAM L97G/C89T

Beam line

ID23-1

ID23-1

Data collection

Resolution $(\AA)^{a}$

$47.45-2.20(2.32-2.20)$

$47.38-1.95(2.06-1.95)$

Space group

C2

C2

Cell dimensions

$a, b, c(\AA)$
$\beta\left({ }^{\circ}\right)$
Monomers per asymmetric unit
$R_{\text {merge }}{ }^{b}$
Wavelength $(\AA)$
$I / \sigma(l)$
Completeness $(\%)$

$181.5,76.49,120.42$

120.0

$0.072(0.40)$

0.9999

$12.0(3.0)$

99.8 (99.9)

72574 (10588)

$3.4(3.5)$

Multiplicity

Refinement

Resolution $(\AA)$

$\mathrm{R}_{\text {work }}{ }^{c}$

$\mathrm{R}_{\text {free }}{ }^{a}$

R.m.s. deviations

Bond lengths $(\AA)$

Bond angles $\left({ }^{\circ}\right)$

Ramachandran (Molprobity)

Favored (\%)

Outlier (\%)

PDB entry

$\begin{array}{ll}2.20 & 1.95 \\ 0.21 & 0.19 \\ 0.25 & 0.22\end{array}$

$0.01 \quad 0.008$

$\begin{array}{ll}1.36 & 1.21\end{array}$

${ }^{a}$ Values in parentheses are for the highest resolution shell.

${ }^{b} R_{\text {merge }}=\Sigma_{\mathrm{h}} \Sigma_{\mathrm{i}}\left|/(\mathrm{h})_{\mathrm{i}}-\langle/(\mathrm{h})\rangle\right| / \Sigma_{\mathrm{h}} \Sigma_{\mathrm{i}}\left\langle/(\mathrm{h})_{\mathrm{i}}\right\rangle$, where $/$ is the observed intensity and \langle\rangle is the average intensity of multiple observations of symmetry-related reflections.

${ }^{c} R_{\text {work }}=\Sigma_{\mathrm{h}}\left|\mathrm{F}_{\text {obs }}-\mathrm{F}_{\text {calc }} / / \Sigma_{\mathrm{h}}\right| \mathrm{F}_{\text {obs }} \mid$, where $\mathrm{F}_{\text {obs }}$ and $\mathrm{F}_{\text {calc }}$ are the observed and calculated structure factor amplitudes, respectively.

${ }^{d} R_{\text {free }}$ is calculated as $R_{\text {work }}$ using $5 \%$ of all reflections randomly chosen and excluded from structure factor calculation and refinement.

Table S6. Summary of the MIO-Tyr80 COM distances observed at the energy minima of the PMF profiles and at the resting state of simulated PAM variants. Supplementary to Figure $\mathbf{5 c}$.

\begin{tabular}{l|cc|cc}
\hline & \multicolumn{2}{|c|}{ PMF } & \multicolumn{2}{c|}{ Resting } \\
PAM system & $\begin{array}{c}\text { MIO-Tyr80 } \\
\text { distance [nm] }\end{array}$ & s.d. $^{a}$ & $\begin{array}{c}\text { MIO-Tyr80 } \\
\text { distance [nm] }\end{array}$ & s.d. $^{b}$ \\
\hline Wt & 1.27 & 0.03 & 1.27 & 0.02 \\
L97G & 1.31 & 0.03 & 1.32 & 0.02 \\
L97G/C89T & 1.39 & 0.04 & 1.29 & 0.03 \\
L97G/A77T/179S/C89T & 1.41 & 0.03 & 1.36 & 0.03 \\
\hline${ }^{a}$ standard deviation from 1 simulation of 20 ns \\
${ }^{b}$ standard deviation from 47 independent simulations (20 ns) at different \\
random initial velocities
\end{tabular}




\section{Supplementary Methods and Text}

1. Protein expression and purification. Wt PAM and mutants were expressed and purified according to $\mathrm{Wu}$ et al., with the following exceptions. ${ }^{3}$ The expression culture was induced with arabinose at $0.02 \%$ (w/v) and then incubated for $48 \mathrm{~h}$ at $17^{\circ} \mathrm{C}$. Gravity-flow chromatography using a $25 \mathrm{~mL}$ column (Bio-Rad) and $1 \mathrm{~mL}$ of Cobalt resin (Talon) was used for purification. The column was pre-equilibrated with the re-suspension buffer described by $\mathrm{Wu}$ et al. . The lysate was incubated $\left(1 \mathrm{~h}, 4^{\circ} \mathrm{C}\right)$ with the binding resin using an orbital shaker. The bound resin was washed with re-suspension buffer $(24 \mathrm{~mL})$ and then eluted with elution buffer $(4 \mathrm{~mL})$. The elution fraction was concentrated down to $\sim 500 \mu \mathrm{l}$ with a centrifugal filter unit (Amicon, catalog \# UFC903024) and exchanged with storage buffer through three rounds of dilution (14 mL storage buffer) and concentration (down to $\sim 500 \mu \mathrm{l}$ ) using the same centrifugal filter unit $(4,000 \mathrm{rpm}$ ). Total protein content was measured using the Uptima BC assay protein quantification kit (Interchim) and yielded 5-10 mg mL of purified enzyme.

2. HPLC analysis of lyase/mutase activity ratios. For the mutase vs. lyase activity measurements (Figure 2) at a single temperature $\left(30^{\circ} \mathrm{C}, 300 \mathrm{rpm}\right.$ using $1.5 \mathrm{~mL}$ tube shaker (Eppendorf Thermomixer comfort)) using reverse-phase HPLC, the deamination conversions of $(R)$ - $\beta$-phenylalanine were measured by detecting the elution analytes at $\lambda_{210}$. The reaction mixture $(2 \mathrm{~mL})$ contained Tris- $\mathrm{HCl}$ buffer $(20 \mathrm{mM}, \mathrm{pH} 8.5,50 \mathrm{mM} \mathrm{NaCl})$, purified PAM enzyme variant $\left(100 \mu \mathrm{g} \mathrm{mL}^{-1}\right.$ final conc.), and $5 \mathrm{mM}$ substrate. For the amination reaction using trans-cinnamic acid $(1 \mathrm{mM})$ as substrate, ammonia solution $(6 \mathrm{M}, \mathrm{pH} 10)$ was added to the substrate, followed by addition of purified PAM enzyme variant $\left(500 \mu \mathrm{g} \mathrm{mL}^{-1}\right.$ final conc) to give a final reaction volume of $2 \mathrm{~mL}$. At each time point $(5,10,15,20,25,30,40,60,120$, and 1,900 min) for both amination and deamination reactions, $100 \mu \mathrm{L}$ samples were taken and heat inactivated for $5 \mathrm{~min}$ at $96^{\circ} \mathrm{C}$ to denature the enzyme. After centrifugation $\left(10 \mathrm{~min}, 16,000 \mathrm{~g}, 4^{\circ} \mathrm{C}\right)$, the supernatant was analyzed using an Alltech $\mathrm{C} 183 \mu$ column (Ridderkerk; $3 \mu \mathrm{m}, 100 \mathrm{~mm} \times 4.6 \mathrm{~mm}$ ) to separate $\alpha$-phenylalanine, $\beta$-phenylalanine, and cinnamic acid. Kphosphate buffer ( $50 \mathrm{mM}, \mathrm{pH} 3$, eluent A) was used for stationary phase and HPLC-grade acetonitrile (eluent B)

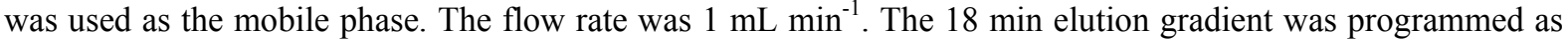
follows: start at $97 \%$ A for $7 \mathrm{~min}, 2 \mathrm{~min}$ to $94 \% \mathrm{~A}, 2.5 \mathrm{~min}$ to $30 \% \mathrm{~A}, 30 \% \mathrm{~A}$ for $1 \mathrm{~min}, 0.5 \mathrm{~min}$ to $97 \% \mathrm{~A}, 5$ min at $97 \% \mathrm{~A}$. The concentrations of $\alpha$-phenylalanine, $\beta$-phenylalanine, and cinnamic acid were calculated from the peak areas based on corresponding standard curves.

For the mutase vs. lyase activity measurements (Figure 4) at multiple temperatures $(15,30,40,50,60$, and $70^{\circ} \mathrm{C}$ ) using the above reverse-phase HPLC system, the deamination conversion of $(R)-\beta$-phenylalanine to $(S)$ - $\alpha$-phenylalanine (mutase product) and trans-cinnamic acid (lyase product) was measured as described above, with a few modifications: (1) reaction volume, $1.4 \mathrm{~mL}$; (2) sample time points (min.): 5, 10, 15, 20, 30, $60,90,120,240,480$, and 1440); and (3) 500 rpm shaking.

3. Chiral HPLC analysis of amination products. The enantioselectivity of the relevant PAM loop mutants (footnote b,Table 1) was analyzed using a reverse phase HPLC chiral column (Daicel Chemical Ind. LTD.: Crownpak CR(+), $4 \mathrm{~mm} \times 150 \mathrm{~mm}, 5 \mu \mathrm{m}$ particle size) at $-6^{\circ} \mathrm{C}$ with aqueous $\mathrm{HClO}_{4}, 8 \% \mathrm{MeOH}, \mathrm{pH} 2.0$ as the mobile phase at a flow rate of $0.2 \mathrm{~mL} \mathrm{~min}^{-1}$ isocratically for $140 \mathrm{~min}$ per run. A UV detector at $210 \mathrm{~nm}$ was used to measure the elution profiles of the analytes. The average retention times were: $41.1 \mathrm{~min}(R)$ - $\alpha$ phenylalanine, $78.6 \mathrm{~min}(S)$ - $\alpha$-phenylalanine, $97.5 \mathrm{~min}(S)$ - $\beta$-phenylalanine, and $119.3 \mathrm{~min}(R)$ - $\beta$-phenylalanine. The concentrations were calculated from the peak areas based on corresponding standard curves. The amination reactions $(200 \mu \mathrm{L}$ total volume, $5 \mathrm{mM} t$-CA as substrate) for the relevant PAM loop mutants were performed at $30^{\circ} \mathrm{C}$ (300 rpm shaking using an Eppendorf ThermomixerC) and a $20 \mu \mathrm{L}$ sample was used for analysis. The remaining sample work-up was followed according to Wu et al.. ${ }^{3}$

4. In silico methods: pulling and umbrella-sampling simulations. Subsequent to the experimental work, MD simulations using an umbrella-sampling approach were performed in order to estimate the free energy differences for disengaging the respective inner loops of various PAM loop mutants (Figure 5, Supplementary Figure S4), as well as quantifying the buried hydrophobic surface area (Figure S5), and secondary structural features within this loop region (Figure 6). Wt TcPAM was used as the starting model for all simulated PAM systems since its structure is known (PDB-ID: 2YII ${ }^{2}$ ), its gene template was used for mutagenesis, and complete structural information for all loops are available. The mutants considered in this in silico study are denoted as L97G, L97G/C89T and L97G/A77T/I79S/C89T. The Wt structure was used as a control. The $\mathrm{N}$ - and $\mathrm{C}$-termini of each protein monomer was modeled in its charged forms. All other titratable amino acids were assigned their canonical state at physiological $\mathrm{pH}$. 
Parameters from the standard GROMOS96 53A6 force field were applied to all components of the simulated systems. The necessary parameters for the MIO cofactor were generated by the Automatic Topology Builder (ATB) website for the above-mentioned force field. ${ }^{4}$ Improper dihedrals were manually adjusted to assure ring planarity of the MIO cofactor. Short-range non-bonded interactions were cut off at $1.4 \mathrm{~nm}$, with long-range electrostatics calculated using the particle mesh Ewald (PME) algorithm. ${ }^{5}$ Dispersion correction was applied to energy and pressure terms to account for truncation of van der Waals terms. Periodic boundary conditions were applied in all directions.

In order to generate equilibrated starting structures for the pulling simulations, each system (Wt and mutants) was placed in a dodecahedral box (with dimensions sufficient to satisfy the minimum image convention and provide space for the pulling of the loop) of simple point charge (SPC) water, to which $100 \mathrm{mM}$ $\mathrm{NaCl}$ (including neutralizing counter-ions) was added to simulate physiological salinity. ${ }^{6}$ Following steepest descents minimization (50000 steps, or maximum force lower than $100 \mathrm{~kJ} \mathrm{~mol}^{-1} \mathrm{~nm}^{-2}$ ), each of the systems were equilibrated in two steps, with position restraints applied to the protein heavy atoms throughout. The first phase involved simulating for $100 \mathrm{ps}$ under a constant volume (NVT) ensemble. The complete system was coupled to temperature coupling baths, and temperature was maintained at $298 \mathrm{~K}$ using the V-rescale coupling method. ${ }^{7}$ Following NVT equilibration, 500 ps of constant pressure (NPT) equilibration were performed, also using weak coupling to maintain pressure isotropically at 1.0 bar. $^{8}$

Pre-pulling MD simulations were conducted for $10 \mathrm{~ns}$ in the presence of position restraints for all $\alpha$ carbon atoms to assure side-chain equilibration but not backbone movements, especially of the inner loop regions. For this pre-pulling simulation, the Nosé-Hoover thermostat was used to maintain temperature, and the Parrinello-Rahman barostat was used to isotropically regulate pressure. ${ }^{9-11}$ This combination of thermostat and barostat ensures that a true NPT ensemble is sampled. Structures from the end of each of these trajectories (Wt, L97G, L97G/C89T and L97G/A77T/I79S/C89T) were used as starting configurations for pulling simulations. Only the loops of chain A were submitted to the pulling protocol described here, while chains B-D were allowed to fully relax during the execution of the pulling. Following equilibration, restraints were removed from all protein chains except for the atoms forming the MIO cofactor of chain A, whose center of mass was used as reference for the pulling simulations. For each of the systems studied here, the center of mass of the catalytic residue Tyr 80 of chain A was pulled away from the MIO cofactor allowing full mobility in all directions over $500 \mathrm{ps}$, using a spring constant of $1000 \mathrm{~kJ} \mathrm{~mol}^{-1} \mathrm{~nm}^{-2}$ and a pull rate of $0.01 \mathrm{~nm} \mathrm{ps}^{-1}$. A final center of mass (COM) distance between peptides A and B of approximately $5.5 \mathrm{~nm}$ was achieved.

From these trajectories, snapshots were taken to generate the starting configurations for the umbrella sampling windows. ${ }^{12} \mathrm{~A}$ spacing of $0.1 \mathrm{~nm}$ COM separation was used, resulting in 47 windows. Each window was submitted to a $20 \mathrm{~ns} \mathrm{MD}$ simulation in order to produce the production run for umbrella sampling analysis. All simulations were conducted using the GROMACS package, version 4.5.5. ${ }^{13}$ The analysis of the simulations was performed using the analysis tools provided in the GROMACS package. The DSSP database was used for secondary structure analyses and the g_sas utility in the GROMACS package was used for SASA calculations. 14,15

All molecular graphical presentations were created by VMD or UCSF Chimera packages. ${ }^{16,17}$ The potential of mean force (PMF) analysis of umbrella sampling runs was performed with the weighted histogram analysis method (WHAM), while its error analysis was conducted using a bootstrap method described previously. ${ }^{18,19}$ Statistical analyses were carried out utilizing ANOVA procedure together with Bonferroni's multiple-comparison test using GraphPad Prism version 6.0 for Mac, GraphPad Software (www.graphpad.com).

4a. Solvent accessible surface area (SASA) calculations. The total SASA $\%$ decrease $(-4 \pm 1 \%)$ for the quadruple mutant comprises drastic changes in both the hydrophilic ( $4 \pm 2 \%$ increase) and hydrophobic (12 $\pm 1 \%$ decrease) SASA (Figure S5). Compared to the quadruple mutant, PAM L97G displayed a slightly less decrease $(-3 \pm 1 \%)$ in total SASA \% that is mostly influenced by a decrease $(-6 \pm 1 \%)$ in hydrophobic SASA. The total SASA \% change for the double loop mutant seems to be mostly dictated by the decreased hydrophobic SASA $(-3 \pm 2 \%)$.

4b. Secondary structure analyses of the PAM inner loop. Figures $6 \mathrm{~b}-\mathbf{e}$ show that the percentage of residues in coil, $\alpha$-helix, turn, $\beta$-bridge, $3_{10}$-helix, bend, and $\beta$-ladder conformations per residue are present for the same inner loop region analyzed in panel A. From panel B, it is apparent that a Gly mutation at position 97 increased the amount of coil conformation at this specific point. Also, it is important to note that the Wt, L97G, and L97G/C89T systems contain $3_{10}$-helix clusters (residues 87-89) that seem to be in equilibrium with turn and bend conformations. On the contrary, the quadruple mutant showed a significant loss of $3_{10}$-helix conformation in this region. The cluster seems to be flanked by two extended portions of $\beta$-ladder-rich conformations (residues 84-86 and 89-93). PAM L97G and L97G/C89T show a small increase in $\beta$-ladder conformation in these portions, which possibly stabilizes the formation of the $33_{10}$-helix cluster. 


\section{References}

(1) Bartsch, S., Wybenga, G. G., Jansen, M., Heberling, M. M., Wu, B., Dijkstra, B. W., and Janssen, D. B. (2013) Redesign of a phenylalanine aminomutase into a phenylalanine ammonia lyase. ChemCatChem 5, 1797-1802.

(2) Wu, B., Szymański, W., Wybenga, G. G., Heberling, M. M., Bartsch, S., de Wildeman, S., Poelarends, G. J., Feringa, B. L., Dijkstra, B. W., and Janssen, D. B. (2012) Mechanism-inspired engineering of phenylalanine aminomutase for enhanced $\beta$ regioselective asymmetric amination of cinnamates. Angew. Chemie 124, 497-501.

(3) Wu, B., Szymański, W., Wietzes, P., de Wildeman, S., Poelarends, G. J., Feringa, B. L., and Janssen, D. B. (2009) Enzymatic synthesis of enantiopure $\alpha$ - and $\beta$-amino acids by phenylalanine aminomutase-catalysed amination of cinnamic acid derivatives. ChemBioChem 10, 338-344.

(4) Malde, A. K., Zuo, L., Breeze, M., Stroet, M., Poger, D., Nair, P. C., Oostenbrink, C., and Mark, A. E. (2011) An automated force field topology builder (ATB) and repository: version 1.0. J. Chem. Theory Comput. 7, 4026-4037.

(5) Darden, T., York, D., and Pedersen, L. (1993) Particle mesh Ewald: An N•log(N) method for Ewald sums in large systems. J. Chem. Phys. 98, 10089-10092.

(6) Berendsen, H. J. C., Postma, J. P. M., Gunsteren, W. F., and Hermans, J. (1981) Interaction models for water in relation to protein hydration. Jerusalem Symp. Quantum Chem. Biochem. 14, 331-342.

(7) Bussi, G., Donadio, D., and Parrinello, M. (2007) Canonical sampling through velocity rescaling. J. Chem. Phys. 126, 14101.

(8) Berendsen, H. J. C., Postma, J. P. M., van Gunsteren, W. F., DiNola, A., and Haak, J. R. (1984) Molecular dynamics with coupling to an external bath. J. Chem. Phys. 81, 3684.

(9) Parrinello, M. (1981) Polymorphic transitions in single crystals: A new molecular dynamics method. J. Appl. Phys. 52, 7182.

(10) Hoover, W. (1985) Canonical dynamics: Equilibrium phase-space distributions. Phys. Rev. A 31, 1695-1697.

(11) Nosé, S. (1984) A unified formulation of the constant temperature molecular dynamics methods. J. Chem. Phys. 81, 511.

(12) Torrie, G. M., and Valleau, J. P. (1977) Nonphysical sampling distributions in Monte Carlo free-energy estimation: Umbrella sampling. J. Comput. Phys. 23, 187-199.

(13) Hess, B., Kutzner, C., van der Spoel, D., and Lindahl, E. (2008) GROMACS 4: Algorithms for highly efficient, loadbalanced, and scalable molecular simulation. J. Chem. Theory Comput. 4, 435-447.

(14) Joosten, R. P., te Beek, T. A. H., Krieger, E., Hekkelman, M. L., Hooft, R. W. W., Schneider, R., Sander, C., and Vriend, G. (2011) A series of PDB related databases for everyday needs. Nucleic Acids Res. 39, D411-419.

(15) Eisenhaber, F., Lijnzaad, P., Argos, P., Sander, C., and Scharf, M. (1995) The double cubic lattice method: Efficient approaches to numerical integration of surface area and volume and to dot surface contouring of molecular assemblies. J. Comput. Chem. 16, 273-284.

(16) Humphrey, W., Dalke, A., and Schulten, K. (1996) VMD: Visual molecular dynamics. J. Mol. Graph. 14, 33-38.

(17) Pettersen, E. F., Goddard, T. D., Huang, C. C., Couch, G. S., Greenblatt, D. M., Meng, E. C., and Ferrin, T. E. (2004) UCSF Chimera-a visualization system for exploratory research and analysis. J. Comput. Chem. 25, 1605-1612.

(18) Kumar, S., Rosenberg, J. M., Bouzida, D., Swendsen, R. H., and Kollman, P. A. (1992) THE weighted histogram analysis method for free-energy calculations on biomolecules. I. The method. J. Comput. Chem. 13, 1011-1021.

(19) Hub, J. S., and de Groot, B. L. (2006) Does $\mathrm{CO}_{2}$ permeate through aquaporin-1? Biophys. J. 91, 842-848. 